\title{
ON THE DESCENT POLYNOMIAL OF SIGNED MULTIPERMUTATIONS
}

\author{
ZHICONG LIN
}

(Communicated by Jim Haglund)

\begin{abstract}
Motivated by a conjecture of Savage and Visontai about the equidistribution of the descent statistic on signed permutations of the multiset $\{1,1,2,2, \ldots, n, n\}$ and the ascent statistic on $(1,4,3,8, \ldots, 2 n-1,4 n)$ inversion sequences, we investigate the descent polynomial of the signed permutations of a general multiset (multipermutations). We obtain a factorial generating function formula for a $q$-analog of these descent polynomials and apply it to show that they have only real roots. Two different proofs of the conjecture of Savage and Visontai are provided. Furthermore, multivariate identities that enumerate two different Euler-Mahonian distributions on type B Coxeter groups due to Beck and Braun are generalized to signed multipermutations.
\end{abstract}

\section{INTRODUCTION}

For each sequence of positive integers $\mathbf{s}=\left\{s_{i}\right\}_{i \geq 1}$, let $\mathfrak{I}_{n}^{(\mathbf{s})}$ be the set of $\mathbf{s}$ inversion sequences of length $n$ defined as

$$
\mathfrak{I}_{n}^{(\mathbf{s})}:=\left\{\left(e_{1}, \ldots, e_{n}\right) \in \mathbb{Z}^{n}: 0 \leq e_{i}<s_{i} \text { for } 1 \leq i \leq n\right\} .
$$

The ascent set of an s-inversion sequence $\mathbf{e}=\left(e_{1}, \ldots, e_{n}\right) \in \mathfrak{I}_{n}^{(\mathbf{s})}$ is the set

$$
\operatorname{Asc}(\mathbf{e}):=\left\{0 \leq i<n: \frac{e_{i}}{s_{i}}<\frac{e_{i+1}}{s_{i+1}}\right\},
$$

with the convention that $e_{0}=0$ and $s_{0}=1$. Let $\operatorname{asc}(\mathbf{e}):=|\operatorname{Asc}(\mathbf{e})|$ be the ascent statistic on $\mathbf{e} \in \mathfrak{I}_{n}^{(\mathbf{s})}$. The $\mathbf{s}$-inversion sequences and the ascent statistic were introduced by Savage and Schuster in [13]. When $\mathbf{s}=(1,2,3, \ldots)$, the set of $\mathbf{s}-$ inversion sequences $\mathfrak{I}_{n}^{(\mathbf{s})}$ is known as the set of inversion tables (or Lehmer codes) of length $n$.

Let $\pi=\pi_{1} \pi_{2} \cdots \pi_{n}$ be a word of length $n$ with letters from $\mathbb{Z}$. A descent of $\pi$ is an index $i \in\{0,1, \ldots, n-1\}$ such that $\pi_{i}>\pi_{i+1}$ (with the convention that $\left.\pi_{0}=0\right)$. Denote by $\operatorname{DES}(\pi)$ the set of descents of $\pi$ and by $\operatorname{des}(\pi)$ the number of descents of $\pi$. The major index of $\pi$, denoted maj $(\pi)$, is defined as

$$
\operatorname{maj}(\pi):=\sum_{i \in \operatorname{DES}(\pi)} i
$$

Received by the editors November 2, 2013 and, in revised form, February 13, 2014, March 12, 2014 and June 4, 2014.

2010 Mathematics Subject Classification. Primary 05A05, 05A15, 01A19.

Key words and phrases. Descents, ascents, inversion sequences, signed multipermutations, realrootedness. 
In this paper, permutations or signed permutations of a multiset are viewed as words on $\mathbb{Z}$.

It is well known that the descent number on permutations of $[n]:=\{1,2, \ldots, n\}$ has the same distribution as the ascent number on inversion tables of length $n$; see [13, Lemma 1] for multivariate equidistribution. Let $P(\{1,1,2,2, \ldots, n, n\})$ be the set of permutations of the multiset $\{1,1,2,2, \ldots, n, n\}$. The following connection between multiset permutations and special inversion sequences was shown in [14, Theorem 3.23].

Theorem 1 (Savage-Visontai [14]).

$$
\sum_{\pi \in P(\{1,1,2,2, \ldots, n, n\})} t^{\operatorname{des}(\pi)}=\sum_{\mathbf{e} \in \mathfrak{I}_{2 n}^{(1,1,3,2, \ldots, 2 n-1, n)}} t^{\operatorname{asc}(\mathbf{e})} .
$$

Now, we consider the signed multiset permutations. Let $P^{ \pm}(\{1,1,2,2, \ldots, n, n\})$ be the set of all signed permutations of the multiset $\{1,1,2,2, \ldots, n, n\}$, whose elements are those of the form $\pm \pi_{1} \pm \pi_{2} \cdots \pm \pi_{2 n}$ with $\pi_{1} \pi_{2} \cdots \pi_{2 n} \in P(\{1,1,2,2, \ldots$, $n, n\})$. For convenience, write $-n$ by $\bar{n}$ for each positive integer $n$. For example,

$$
P^{ \pm}(\{1,1\})=\{11,1 \overline{1}, \overline{1} 1, \overline{1} \overline{1}\} .
$$

Clearly,

$$
\left|P^{ \pm}(\{1,1,2,2, \ldots, n, n\})\right|=\frac{(2 n) !}{2^{n}} 2^{2 n}=2^{n}(2 n) !=\left|\mathfrak{I}_{2 n}^{(1,4,3,8, \ldots, 2 n-1,4 n)}\right| .
$$

Savage and Visontai [14, Conjecture 3.25] further conjectured the following equidistribution, which was proved very recently (and independently) by Chen et al. [4] using type B P-Partitions.

Theorem 2. For any $n \geq 1$, we have

$$
\sum_{\pi \in P^{ \pm}(\{1,1,2,2, \ldots, n, n\})} t^{\operatorname{des}(\pi)}=\sum_{\mathbf{e} \in \mathfrak{I}_{2 n}^{(1,4,3,8, \ldots, 2 n-1,4 n)}} t^{\operatorname{asc}(\mathbf{e})} .
$$

For $n \leq 2$, we have

$$
\sum_{\pi \in P^{ \pm}(\{1,1\})} t^{\operatorname{des}(\pi)}=1+3 t=\sum_{\mathbf{e} \in \mathfrak{I}_{2}^{(1,4)}} t^{\operatorname{asc}(\mathbf{e})}
$$

and

$$
\sum_{\pi \in P^{ \pm}(\{1,1,2,2\})} t^{\operatorname{des}(\pi)}=1+31 t+55 t^{2}+9 t^{3}=\sum_{\mathbf{e} \in \mathfrak{I}_{4}^{(1,4,3,8)}} t^{\operatorname{asc}(\mathbf{e})} .
$$

In Section 2, we will give an elementary proof of Theorem 2 by verifying that both sides of (1.1) satisfy the same recurrence formula. Motivated by this conjecture, in the rest of this paper, we study the descent polynomial of signed permutations of a general multiset (or called general signed multipermutations for brevity). In Section 3 , we prove a factorial generating function formula for the (des, fmaj)enumerator of general signed multipermutations, which generalizes a result of Chow and Gessel [5]. In particular, a different proof of Theorem 2 is also provided. In Section 4, we use the factorial formula to show that the descent polynomial of the signed multipermutations is real-rooted. Moreover, another different descent polynomial of the signed multipermutations is also shown to be real-rooted. In Section [5, we extend some multivariate identities due to Beck and Braun [1] from 
permutations to (signed) multipermutations. We end this paper with some related open questions.

\section{Proof of Theorem 2}

Lemma 3. Let

$$
E_{n}(t)=\sum_{i=0}^{2 n-1} E_{n, i} t^{i}:=\sum_{\mathbf{e} \in \mathfrak{I}_{2 n}^{(1,4,3,8, \ldots, 2 n-1,4 n)}} t^{\operatorname{asc}(\mathbf{e})} .
$$

Then,

$E_{n+1, i}=\left(2 i^{2}+3 i+1\right) E_{n, i}+(2 i(4 n-2 i+3)+2 n+1) E_{n, i-1}+(2 n+2-i)(4 n-2 i+5) E_{n, i-2}$, with boundary conditions $E_{n, i}=0$ for $i<0$ or $i>2 n-1$.

Proof. The following formula was established in [13. Theorem 13] using Ehrhart theory:

$$
\frac{E_{n}(t)}{(1-t)^{2 n+1}}=\sum_{k \geq 0}((k+1)(2 k+1))^{n} t^{k} .
$$

Thus we have

$$
\begin{aligned}
\frac{E_{n+1}(t)}{(1-t)^{2 n+3}} & =\sum_{k \geq 0}((k+1)(2 k+1))^{n+1} t^{k} \\
& =\sum_{k \geq 0}((k+1)(2 k+1))^{n}(k+1)(2 k+1) t^{k} \\
& =\sum_{k \geq 0}((k+1)(2 k+1))^{n}(2 k(k-1)+5 k+1) t^{k} \\
& =2 t^{2}\left(E_{n}(t)(1-t)^{-2 n-1}\right)^{\prime \prime}+5 t\left(E_{n}(t)(1-t)^{-2 n-1}\right)^{\prime}+\frac{E_{n}(t)}{(1-t)^{2 n+1}} .
\end{aligned}
$$

Multiplying both sides by $(1-t)^{2 n+3}$ gives

$$
\begin{aligned}
E_{n+1}(t)= & 2 t^{2}\left[E_{n}^{\prime \prime}(t)+\left[(8 n+4) t^{2}(1-t)+5 t(1-t)^{2}\right] E_{n}^{\prime}(t)\right. \\
& +\left[\left(8 n^{2}+2 n\right) t^{2}+(10 n+3) t+1\right] E_{n}(t) .
\end{aligned}
$$

Extracting the coefficients of $t^{i}$ on both sides, we have

$$
\begin{aligned}
E_{n+1, i}= & 2 i(i-1) E_{n, i}-4(i-1)(i-2) E_{n, i-1}+2(i-2)(i-3) E_{n, i-2} \\
& +5 i E_{n, i}+(8 n-6)(i-1) E_{n, i-1}+(1-8 n)(i-2) E_{n, i-2} \\
& +E_{n, i}+(10 n+3) E_{n, i-1}+\left(8 n^{2}+2 n\right) E_{n, i-2},
\end{aligned}
$$

which becomes (2.1) after simplification.

Lemma 4. Let

$$
P_{n}(t)=\sum_{i=0}^{2 n-1} P_{n, i} t^{i}:=\sum_{\pi \in P^{ \pm}(\{1,1,2,2, \ldots, n, n\})} t^{\operatorname{des}(\pi)} .
$$

Then,

$P_{n+1, i}=\left(2 i^{2}+3 i+1\right) P_{n, i}+(2 i(4 n-2 i+3)+2 n+1) P_{n, i-1}+(2 n+2-i)(4 n-2 i+5) P_{n, i-2}$, with boundary conditions $P_{n, i}=0$ for $i<0$ or $i>2 n-1$. 
Proof. Denote by $\mathcal{P}_{n, i}$ the set of signed permutations of $\{1,1,2,2, \ldots, n, n\}$ with $i$ descents. Clearly, every signed permutation in $\mathcal{P}_{n+1, i}$ can be obtained from a signed permutation in $\mathcal{P}_{n, i}, \mathcal{P}_{n, i-1}$ or $\mathcal{P}_{n, i-2}$ by inserting $\{n+1, n+1\},\{\overline{n+1}, \overline{n+1}\}$ or $\{n+1, \overline{n+1}\}$.

For each $\pi \in \mathcal{P}_{n, i}$, there are $i+1$ positions in $\pi$ that inserting $n+1$ will not produce an extra descent. These are the $i$ descent positions of $\pi$ plus the position to the right of $\pi$. Differently, we can only insert $\overline{n+1}$ to the $i$ descent positions of $\pi$ that may not produce an extra descent. Therefore, there are $\left(\left(\begin{array}{c}i+1 \\ 2\end{array}\right)\right)=\left(\begin{array}{c}i+2 \\ 2\end{array}\right)$ ways to insert $\{n+1, n+1\},\left(\left(\begin{array}{c}i \\ 2\end{array}\right)\right)=\left(\begin{array}{c}i+1 \\ 2\end{array}\right)$ ways to insert $\{\overline{n+1}, \overline{n+1}\}$ and $(i+1) i$ ways to insert $\{n+1, \overline{n+1}\}$ into $\pi$ to let it become a signed permutation in $\mathcal{P}_{n+1, i}$. So there are $\left(2 i^{2}+3 i+1\right) P_{n, i}$ signed permutations in $\mathcal{P}_{n+1, i}$ constructed from $\mathcal{P}_{n, i}$.

Similarly, there are $(2 i(4 n-2 i+3)+2 n+1) P_{n, i-1}$ and $(2 n+2-i)(4 n-2 i+5) P_{n, i-2}$ signed permutations in $\mathcal{P}_{n+1, i}$ constructed from $\mathcal{P}_{n, i-1}$ and $\mathcal{P}_{n, i-2}$, respectively.

Summarizing all the above three cases, we obtain (2.3).

By Lemmas 3 and 4 , we see that $E_{n, i}$ and $P_{n, i}$ satisfy the same recurrence relation and boundary conditions, so they are equal. This finishes the proof of Theorem 2 .

\section{General signed multipermutations}

In this section, we consider the descent polynomial on signed permutations of the general multiset $M_{\mathbf{m}}:=\left\{1^{m_{1}}, 2^{m_{2}}, \ldots, n^{m_{n}}\right\}$ for each vector $\mathbf{m}:=\left(m_{1}, m_{2}, \ldots, m_{n}\right)$ $\in \mathbb{P}^{n}$. Let $P(\mathbf{m})$ and $P^{ \pm}(\mathbf{m})$ denote the set of all permutations and signed permutations of the multiset $M_{\mathbf{m}}$, respectively.

Recall that the $q$-shifted factorial $(t ; q)_{n}$ is defined by $(t ; q)_{n}:=\prod_{i=0}^{n-1}\left(1-t q^{i}\right)$ for any positive integer $n$ and $(t ; q)_{0}=1$. The $q$-binomial coefficient $\left[\begin{array}{l}n \\ k\end{array}\right]_{q}$ is then defined as

$$
\left[\begin{array}{l}
n \\
k
\end{array}\right]_{q}:=\frac{(q ; q)_{n}}{(q ; q)_{n-k}(q ; q)_{k}}
$$

for $n \geq k \geq 0$. It is convenient to use $\left[\begin{array}{c}-1 \\ 0\end{array}\right]_{q}=1$ here. The following result is attributed to MacMahon [11, Volume 2, p. 211], whose proof can be found in [9, § 7].

Theorem 5. For every $\mathbf{m} \in \mathbb{P}^{n}$ with $m_{1}+\cdots+m_{n}=m$, we have

$$
\frac{\sum_{\pi \in P(\mathbf{m})} t^{\operatorname{des}(\pi)} q^{\operatorname{maj}(\pi)}}{(t ; q)_{m+1}}=\sum_{k \geq 0}\left[\begin{array}{c}
m_{1}+k \\
m_{1}
\end{array}\right]_{q} \ldots\left[\begin{array}{c}
m_{n}+k \\
m_{n}
\end{array}\right]_{q} t^{k}
$$

Our signed version is:

Theorem 6. For every $\mathbf{m} \in \mathbb{P}^{n}$ with $m_{1}+\cdots+m_{n}=m$, we have $(3.2)$

$$
\frac{\sum_{\pi \in P^{ \pm}(\mathbf{m})} t^{\mathrm{des}(\pi)} q^{\mathrm{fmaj}(\pi)} z^{\mathrm{neg}(\pi)}}{\left(t ; q^{2}\right)_{m+1}}=\sum_{k \geq 0} \prod_{r=1}^{n}\left(\sum_{i=0}^{m_{r}}(z q)^{i}\left[\begin{array}{c}
m_{r}-i+k \\
m_{r}-i
\end{array}\right]_{q^{2}}\left[\begin{array}{c}
i+k-1 \\
i
\end{array}\right]_{q^{2}}\right) t^{k},
$$

where $\operatorname{fmaj}(\pi):=2 \operatorname{maj}(\pi)+\operatorname{neg}(\pi)$ and $\operatorname{neg}(\pi)$ is the number of negative signs in $\pi$. In particular,

$$
\frac{\sum_{\pi \in P^{ \pm}(\mathbf{m})} t^{\operatorname{des}(\pi)} q^{\mathrm{fmaj}(\pi)}}{\left(t ; q^{2}\right)_{m+1}}=\sum_{k \geq 0} \prod_{r=1}^{n}\left[\begin{array}{c}
m_{r}+2 k \\
m_{r}
\end{array}\right]_{q} t^{k}
$$


Remark 1. Setting $z=0$, we recover Theorem 5] It is worth noticing that Foata and Han [8, Theorem 1.2] has calculated (another signed version of Theorem 5) the factorial generating function formula for

$$
\sum_{\pi \in P^{ \pm}(\mathbf{m})} t^{\mathrm{fdes}(\pi)} q^{\mathrm{fmaj}(\pi)} z^{\mathrm{neg}(\pi)},
$$

involving the so-called flag descent statistic fdes, fdes $(\pi):=2 \operatorname{des}(\pi)-\chi\left(\pi_{1}<0\right)$, on signed multipermutations (or words).

As usual, the $q$-integer $[n]_{q}:=1+q+\cdots+q^{n-1}$ for each $n \geq 1$.

Corollary 7 (Chow-Gessel [5]).

$$
\frac{\sum_{\pi \in P^{ \pm}(\{1,2, \ldots, n\})} t^{\mathrm{des}(\pi)} q^{\mathrm{fmaj}(\pi)}}{\left(t ; q^{2}\right)_{n+1}}=\sum_{k \geq 0}[2 k+1]_{q}^{n} t^{k} .
$$

Proof. Setting $m_{1}=\cdots=m_{n}=1$ and $z=1$ in Theorem 6 .

\section{Corollary 8.}

$$
\frac{\sum_{\pi \in P^{ \pm}(\{1,1,2,2, \ldots, n, n\})} t^{\operatorname{des}(\pi)}}{(1-t)^{2 n+1}}=\sum_{k \geq 0}((k+1)(2 k+1))^{n} t^{k} .
$$

Proof. Setting $m_{1}=\cdots=m_{n}=2$ and $z=q=1$ in Theorem 6

Remark 2. Comparing (3.4) with (2.2) we get another proof of Theorem 2 ,

We will prove Theorem 6 by using the technique of barred permutations inspired by Gessel and Stanley [7. For each $\pi=\pi_{1} \cdots \pi_{m} \in P^{ \pm}(\mathbf{m})$, we call the space between $\pi_{i}$ and $\pi_{i+1}$ the $i$-th space of $\pi$ for $0<i<m$. We also call the space before $\pi_{1}$ and the space after $\pi_{m}$ the 0 -th space and the $m$-th space of $\pi$, respectively. If $i \in \operatorname{DES}(\pi)$, then we call the $i$-th space a descent space.

A barred permutation on $\pi \in P^{ \pm}(\mathbf{m})$ is obtained by inserting one or more vertical bars into some spaces of $\pi$ such that there is at least one bar in every descent space of $\pi$. For example, $\| \overline{1}|\overline{2} 2| 1||$ is a barred permutation on $\pi=\overline{1} \overline{2} 21$ but $\overline{1}|\overline{2} 2| 1||$ is not, since $0 \in \operatorname{DES}(\pi)$ and there is no bar in the 0 -th space (i.e. before $\pi_{1}=\overline{1}$ ).

Proof of Theorem 6. Let $B(\mathbf{m})$ be the set of barred permutations on $P^{ \pm}(\mathbf{m})$. Let $\sigma \in B(\mathbf{m})$ be a barred permutation on $\pi$ with $b_{i}$ bars in the $i$-th space of $\pi$. We define the weight $\operatorname{wt}(\sigma)$ to be

$$
\operatorname{wt}(\sigma):=t^{\sum_{i=0}^{m} b_{i}} q^{\operatorname{neg}(\pi)+2 \sum_{i=1}^{m} i b_{i}} z^{\operatorname{neg}(\pi)} .
$$

For example, wt $(|| \overline{1}|\overline{2} 2| 1||)=t^{6} q^{26} z^{2}$. Now, we count the barred permutations in $B(\mathbf{m})$ by the weight "wt" in two different ways. First, fix a permutation $\pi \in$ $P^{ \pm}(\mathbf{m})$, and sum over all barred permutations on $\pi$. Then, fix the number of bars $k$, and sum over all barred permutations with $k$ bars.

Fix a permutation $\pi$, a barred permutation on $\pi$ can be obtained by inserting one bar in each descent space and then inserting any number of bars in all spaces. So counting all the barred permutations on $\pi$ by the weight "wt" gives

$$
t^{\operatorname{des}(\pi)} q^{\mathrm{fmaj}(\pi)} z^{\operatorname{neg}(\pi)}\left(1+t+t^{2}+\cdots\right)\left(1+t q^{2}+\left(t q^{2}\right)^{2}+\cdots\right) \cdots\left(1+t q^{2 m}+\left(t q^{2 m}\right)^{2}+\cdots\right),
$$


which is equal to $\frac{t^{\operatorname{des}(\pi)} q^{\mathrm{fmaj}(\pi)} z^{\mathrm{neg}(\pi)}}{\left(t ; q^{2}\right)_{m+1}}$. This shows that

$$
\sum_{\sigma \in B(\mathbf{m})} \operatorname{wt}(\sigma)=\frac{\sum_{\pi \in P^{ \pm}(\mathbf{m})} t^{\mathrm{des}(\pi)} q^{\mathrm{fmaj}(\pi)} z^{\mathrm{neg}(\pi)}}{\left(t ; q^{2}\right)_{m+1}} .
$$

For a fixed integer $k \geq 0$, let $B_{k}(\mathbf{m})$ be the set of barred permutations in $B(\mathbf{m})$ with $k$ bars. Now, each barred permutation in $B_{k}(\mathbf{m})$ can be constructed by putting $k$ bars in one line and then inserting $m_{r}$ integers from $\{r,-r\}$, for $1 \leq r \leq n$, to the $k+1$ spaces between each two adjacency bars (including the space on the left side and the right side), with the rules that

- all integers between two adjacency bars are in increasing order;

- negative integers cannot be inserted to the left side of all bars.

Observe that inserting a positive (resp. negative) integer to the $(j+1)$-th space (from right to left) of two adjacency bars would contribute the factor $q^{2 j}$ (resp. $(q z) q^{2 j}$ ) to the weight of the resulting barred permutation. Thus, by the well-known interpretation (cf. 9, Proposition 4.1]) of the $q$-binomial coefficient

$$
\left[\begin{array}{c}
n+r \\
n
\end{array}\right]_{q}=\sum_{0 \leq c_{1} \leq c_{2} \cdots \leq c_{n} \leq r} q^{\sum c_{i}}
$$

we have

$$
\sum_{\sigma \in B_{k}(\mathbf{m})} \operatorname{wt}(\sigma)=\prod_{r=1}^{n}\left(\sum_{i=0}^{m_{r}}(z q)^{i}\left[\begin{array}{c}
m_{r}-i+k \\
m_{r}-i
\end{array}\right]_{q^{2}}\left[\begin{array}{c}
i+k-1 \\
i
\end{array}\right]_{q^{2}}\right) t^{k} .
$$

Summing over all $k$ in the above equation and comparing with equation (3.5) we get (3.2). By setting $z=1$ in (3.2) and using Lemma 9 below, we obtain (3.3).

Lemma 9. For any integers $n, k \geq 0$ we have

$$
\left[\begin{array}{c}
k+n \\
n
\end{array}\right]_{q}=\sum_{i=0}^{n} q^{i}\left[\begin{array}{c}
n-i+\lfloor k / 2\rfloor \\
n-i
\end{array}\right]_{q^{2}}\left[\begin{array}{c}
i+\lfloor(k-1) / 2\rfloor \\
i
\end{array}\right]_{q^{2}} .
$$

Proof. It is easy to check that $\frac{1}{(t ; q)_{k+1}}=\left(\frac{1}{\left(t ; q^{2}\right)_{\lfloor(k+2) / 2\rfloor}}\right)\left(\frac{1}{\left(t q ; q^{2}\right)_{\lfloor(k+1) / 2\rfloor}}\right)$ for any $k \geq 0$. It follows then from the $q$-binomial theorem $\frac{1}{(t ; q)_{k+1}}=\sum_{i \geq 0}\left[\begin{array}{c}k+i \\ i\end{array}\right]_{q} t^{i}$ that

$$
\sum_{i \geq 0}\left[\begin{array}{c}
k+i \\
i
\end{array}\right]_{q} t^{i}=\left(\sum_{i \geq 0}\left[\begin{array}{c}
\lfloor k / 2\rfloor+i \\
i
\end{array}\right]_{q^{2}} t^{i}\right)\left(\sum_{i \geq 0}\left[\begin{array}{c}
\lfloor(k-1) / 2\rfloor+i \\
i
\end{array}\right]_{q^{2}} q^{i} t^{i}\right) .
$$

Taking the coefficient of $t^{n}$ on both sides yields (3.7).

\section{Signed versions of Simion's Result about Real-rootedness}

It was shown in [14, Theorem 1.1] that the ascent polynomial $\sum_{\mathbf{e} \in \mathfrak{I}_{2 n}^{(\mathbf{s})}} t^{\operatorname{asc}(\mathbf{e})}$ has only real roots for each $\mathbf{s} \in \mathbb{P}^{n}$. In view of Theorem 2 we have:

Corollary 10. The polynomial $\sum_{\pi \in P^{ \pm}(\{1,1,2,2, \ldots, n, n\})} t^{\operatorname{des}(\pi)}$ has only real roots for any positive integer $n$.

Simion [15, §2] proved that the descent polynomial on the permutations of a general multiset has only real roots. 
Theorem 11 (Simion [15]). The descent polynomial $\sum_{\pi \in P(\mathbf{m})} t^{\operatorname{des}(\pi)}$ has only real roots for every $\mathbf{m} \in \mathbb{P}^{n}$.

We have the following signed version of Simion's result, which generalizes the $m_{1}=m_{2}=\cdots=m_{n}=1$ (i.e., the type B Coxeter group) case of Brenti [3] and Corollary 10.

Theorem 12. The descent polynomial $\sum_{\pi \in P^{ \pm}(\mathbf{m})} t^{\operatorname{des}(\pi)}$ has only real roots for every $\mathbf{m} \in \mathbb{P}^{n}$.

The key point of the proof of the above result lies in the following simple lemma.

Lemma 13. Let

$$
\frac{F_{n}(t)}{(1-t)^{n+1}}=\sum_{k \geq 0} f(k)(a k+b) t^{k} \quad \text { and } \quad \frac{F_{n-1}(t)}{(1-t)^{n}}=\sum_{k \geq 0} f(k) t^{k} .
$$

If $a>0, n>\frac{b}{a}, F_{n-1}(t)$ is a real-rooted polynomial with nonnegative coefficients and $F_{n}(t)$ is a polynomial with nonnegative coefficients, then $F_{n}(t)$ has only real roots.

Proof. Clearly, by (4.1) we have

$$
\frac{F_{n}(t)}{(1-t)^{n+1}}=\sum_{k \geq 0} f(k)(a k+b) t^{k}=b \frac{F_{n-1}(t)}{(1-t)^{n}}+a\left(\frac{F_{n-1}(t)}{(1-t)^{n}}\right)^{\prime} t .
$$

From the above equation we deduce that

$$
F_{n}(t)=((a n-b) t+b) F_{n-1}(t)+a t(1-t) F_{n-1}^{\prime}(t) .
$$

The lemma then follows by applying a result of Brenti [2, Theorem 2.4.5], which was established through some standard argument by using Rolle's theorem.

Proof of Theorem 12, By setting $q=1, z=1$ in equation (3.3), we have

$$
\frac{\sum_{\pi \in P^{ \pm}(\mathbf{m})} t^{\operatorname{des}(\pi)}}{(1-t)^{m+1}}=\sum_{k \geq 0} \prod_{r=1}^{n} \frac{(2 k+1)(2 k+2) \cdots\left(2 k+m_{r}\right)}{m_{r} !} t^{k}
$$

We proceed by induction on $m=m_{1}+m_{2}+\cdots+m_{n}$ using the above formula.

When $m=1$, then $\sum_{\pi \in P^{ \pm}(\{1\})} t^{\operatorname{des}(\pi)}=1+t$ and the result is true. Suppose that $m \geq 2$ and the result is true for $m-1$. We only treat the case $m_{1} \geq 2$, the case $m_{1}=1$ can be handled similarly. Let $\mathbf{m}-\mathbf{e}_{1}:=\left(m_{1}-1, m_{2}, \ldots, m_{n}\right) \in \mathbb{P}^{n}$. By equation (4.2) we have

$$
\frac{\sum_{\pi \in P^{ \pm}(\mathbf{m})} t^{\operatorname{des}(\pi)}}{(1-t)^{m+1}}=\sum_{k \geq 0} f(k) \frac{\left(2 k+m_{1}\right)}{m_{1}} t^{k} \quad \text { and } \quad \frac{\sum_{\pi \in P^{ \pm}\left(\mathbf{m}-\mathbf{e}_{1}\right)} t^{\operatorname{des}(\pi)}}{(1-t)^{m}}=\sum_{k \geq 0} f(k) t^{k},
$$

where $f(k)=\frac{(2 k+1)(2 k+2) \cdots\left(2 k+m_{1}-1\right)}{\left(m_{1}-1\right) !} \prod_{r=2}^{n} \frac{(2 k+1)(2 k+2) \cdots\left(2 k+m_{r}\right)}{m_{r} !}$. Now we can check that all the conditions of Lemma 13 are satisfied (with $n=m, a=\frac{2}{m_{1}}, b=1$ ):

$$
a=\frac{2}{m_{1}}>0, \quad n=m>\frac{m_{1}}{2}=\frac{b}{a},
$$

the polynomial $\sum_{\pi \in P^{ \pm}\left(\mathbf{m}-\mathbf{e}_{1}\right)} t^{\operatorname{des}(\pi)}$ is real-rooted with nonnegative coefficients by induction hypothesis and $\sum_{\pi \in P^{ \pm}(\mathbf{m})} t^{\operatorname{des}(\pi)}$ has nonnegative coefficients. This implies that $\sum_{\pi \in P^{ \pm}(\mathbf{m})} t^{\operatorname{des}(\pi)}$ is real-rooted, which completes the proof of the theorem. 
Remark 3. Note that the above approach is also available for Theorem 11.

Recall that a polynomial $A(t)=\sum_{k=0}^{n} a_{k} t^{k}$ is unimodal if for some $0 \leq j \leq n$ we have $a_{0} \leq a_{1} \leq \cdots \leq a_{j} \geq a_{j+1} \geq a_{n}$ and is logarithmically concave (or logconcave for short) if $a_{i}^{2} \geq a_{i-1} a_{i+1}$ for all $1 \leq i \leq n-1$. The following interesting properties of the descent polynomial of signed multipermutations can be obtained by combining Theorem 12 and [2, Theorem 1.2.1].

Corollary 14. The descent polynomial $\sum_{\pi \in P^{ \pm}(\mathbf{m})} t^{\operatorname{des}(\pi)}$ is log-concave and unimodal for each $\mathbf{m} \in \mathbb{P}^{n}$.

The following is another signed version of Simion's result.

Theorem 15. The flag descent polynomial $\sum_{\pi \in P^{ \pm}(\mathbf{m})} t^{\mathrm{fdes}(\pi)}$ has only real roots for every $\mathbf{m} \in \mathbb{P}^{n}$. In particular, every flag descent polynomial of signed multipermutations is log-concave and unimodal.

Proof. By [8, Theorem 1.2], we can derive that

$$
\frac{\sum_{\pi \in P^{ \pm}(\mathbf{m})} t^{\mathrm{fdes}(\pi)}}{(1-t)\left(1-t^{2}\right)^{m}}=\sum_{k \geq 0}\left(\begin{array}{c}
m_{1}+k \\
m_{1}
\end{array}\right) \cdots\left(\begin{array}{c}
m_{n}+k \\
m_{n}
\end{array}\right) t^{k} .
$$

On the other hand, by setting $q=1$ in Theorem 5 we have

$$
\frac{\sum_{\pi \in P(\mathbf{m})} t^{\operatorname{des}(\pi)}}{(1-t)^{m+1}}=\sum_{k \geq 0}\left(\begin{array}{c}
m_{1}+k \\
m_{1}
\end{array}\right) \cdots\left(\begin{array}{c}
m_{n}+k \\
m_{n}
\end{array}\right) t^{k} .
$$

Comparing with equation (4.3), we obtain

$$
\sum_{\pi \in P^{ \pm}(\mathbf{m})} t^{\mathrm{fdes}(\pi)}=(1+t)^{m} \sum_{\pi \in P(\mathbf{m})} t^{\mathrm{des}(\pi)} .
$$

Now the flag descent polynomial $\sum_{\pi \in P^{ \pm}(\mathbf{m})} t^{\mathrm{fdes}(\pi)}$ is real-rooted follows from the above relation and Theorem 11 .

Remark 4. For $m_{1}=m_{2}=\cdots=m_{n}=1$, the above result was already known to Mongelli [12].

\section{Multivariate identities}

The following well-known identity is due to Carlitz [6]:

$$
\frac{\sum_{\pi \in \mathfrak{S}_{m}} t^{\operatorname{des}(\pi)} q^{\operatorname{maj}(\pi)}}{(t ; q)_{m+1}}=\sum_{k \geq 0}[k+1]_{q}^{m} t^{k},
$$

where $\mathfrak{S}_{m}$ is the symmetric group of order $m$ (or the group of permutations of $[m])$. Let $z_{0}, z_{1}, z_{2}, \ldots$ be a sequence of variables. Recently, Beck and Braun [1, Theorem 4.1] obtained the following elegant multivariate identity that generalizes Carlitz's identity:

$$
\frac{\sum_{\pi \in \mathfrak{S}_{m}} \prod_{i \in \operatorname{DES}(\pi)} z_{0} z_{\pi_{1}} z_{\pi_{2}} \cdots z_{\pi_{i}}}{\prod_{i=0}^{m}\left(1-z_{0} z_{\pi_{1}} z_{\pi_{2}} \cdots z_{\pi_{i}}\right)}=\sum_{k \geq 0} \prod_{i=1}^{m}[k+1]_{z_{i}} z_{0}^{k} .
$$

This identity, as well as many type B versions, were established via the machine of polyhedral geometry in 1 . 
Remark 5. As pointed out in [1, the approach of polyhedral geometry is related to Stanley's theory of P-Partitions and equation (5.2) resembles Corollary 7.1 in [16. The technique of barred permutations is also closely related to the theory of $P$ Partitions, as both were foreshadowed by work of MacMahon [11.

Note that equation (5.1) is the special case $m_{1}=m_{2}=\cdots=m_{n}=1$ of MacMahon's identity in Theorem 5. Our technique of barred permutations used in the proof of Theorem 6 has the advantage that enables us to extend multivariate identity (5.2) to the multipermutations easily as follows.

Theorem 16. For each $\mathbf{m} \in \mathbb{P}^{n}$ with $m_{1}+\cdots+m_{n}=m$, we have

$$
\frac{\sum_{\pi \in P(\mathbf{m})} \prod_{i \in \operatorname{DES}(\pi)} z_{0} z_{\pi_{1}} z_{\pi_{2}} \cdots z_{\pi_{i}}}{\prod_{i=0}^{m}\left(1-z_{0} z_{\pi_{1}} z_{\pi_{2}} \cdots z_{\pi_{i}}\right)}=\sum_{k \geq 0}\left[\begin{array}{c}
m_{1}+k \\
m_{1}
\end{array}\right]_{z_{1}} \ldots\left[\begin{array}{c}
m_{n}+k \\
m_{n}
\end{array}\right]_{z_{n}} z_{0}^{k} .
$$

The proof of the above identity, together with other multivariate generalizations for signed multipermutations involving the descents and the flag descents, will be given in this section.

Proof of Theorem 16. As $P(\mathbf{m}) \subseteq P^{ \pm}(\mathbf{m})$, the definition of barred permutations on $\pi \in P(\mathbf{m})$ is valid. For any barred permutation $\sigma$ on $\pi$ with $b_{i}$ bars in the $i$-th space of $\pi$, let

$$
\mathrm{wt}_{A}(\sigma)=\prod_{i=0}^{m}\left(z_{0} z_{\pi_{1}} \cdots z_{\pi_{i}}\right)^{b_{i}}
$$

Let $A(\mathbf{m})$ denote the set of all barred permutations on $P(\mathbf{m})$. We will count $A(\mathbf{m})$ according to the weight $\mathrm{wt}_{A}$ in two different ways similarly as in the proof of Theorem 6.

Fix $\pi \in P(\mathbf{m})$. Each barred permutation on $\pi$ can be obtained by inserting one bar in each descent space and then inserting any number of bars in all spaces. So counting all the barred permutations on $\pi$ by the weight $\mathrm{wt}_{A}$ gives

$$
\left(\sum_{k \geq 0} z_{0}^{k}\right)\left(\sum_{k \geq 0}\left(z_{0} z_{\pi_{1}}\right)^{k}\right) \cdots\left(\sum_{k \geq 0}\left(z_{0} z_{\pi_{1}} \cdots z_{\pi_{m}}\right)^{k}\right) \prod_{i \in \operatorname{DES}(\pi)} z_{0} z_{\pi_{1}} z_{\pi_{2}} \cdots z_{\pi_{i}}
$$

which is $\frac{\prod_{i \in \mathrm{DES}(\pi)} z_{0} z_{\pi_{1}} z_{\pi_{2}} \cdots z_{\pi_{i}}}{\prod_{i=0}^{m}\left(1-z_{0} z_{\pi_{1}} z_{\pi_{2}} \cdots z_{\pi_{i}}\right)}$. Therefore we have

$$
\sum_{\sigma \in A(\mathbf{m})} \operatorname{wt}_{A}(\sigma)=\frac{\sum_{\pi \in P(\mathbf{m})} \prod_{i \in \operatorname{DES}(\pi)} z_{0} z_{\pi_{1}} z_{\pi_{2}} \cdots z_{\pi_{i}}}{\prod_{i=0}^{m}\left(1-z_{0} z_{\pi_{1}} z_{\pi_{2}} \cdots z_{\pi_{i}}\right)} .
$$

Fix an integer $k \geq 0$ and let $A_{k}(\mathbf{m})$ be the set of barred permutations in $A(\mathbf{m})$ with $k$ bars. Now, each barred permutation in $A_{k}(\mathbf{m})$ can be constructed by putting $k$ bars in one line and then inserting $m_{r}$ copies of $r$, for $1 \leq r \leq n$, to the $k+1$ spaces between each two adjacency bars (including the left and right spaces). Note that all integers inserted to the same space must be in increasing order; also inserting an integer $i$ in the $(j+1)$-th space (from right to left) contributes the factor $z_{i}^{j}$ 
to the weight of the resulting barred permutation. Thus, by the interpretation of $q$-binomial coefficients (3.6) we have

$$
\sum_{\sigma \in A_{k}(\mathbf{m})} \operatorname{wt}_{A}(\sigma)=\left[\begin{array}{c}
m_{1}+k \\
m_{1}
\end{array}\right]_{z_{1}} \ldots\left[\begin{array}{c}
m_{n}+k \\
m_{n}
\end{array}\right]_{z_{n}} z_{0}^{k} .
$$

Summing over all $k$ and comparing with (5.4) we get (5.3).

Setting $z_{0}=t$ and $z_{1}=z_{2}=\cdots z_{n}=q$ in (5.3) we get (3.1). Also, when $m_{1}=\cdots=m_{r}=1$ identity (5.3) becomes the Beck-Braun identity (5.2).

We continue to generalize other Beck-Braun identities of type B to signed multipermutations. For any $\pi=\pi_{1} \cdots \pi_{m} \in P^{ \pm}(\mathbf{m})$, introduce the sign change function $\delta^{\pi}:\{1,2, \cdots, m\} \rightarrow\{0,1\}^{m}$ as

$$
\delta^{\pi}(i)=\delta_{i}^{\pi}:=1 \text { if } \pi_{i} \pi_{i+1}<0 \text { or } i=m \text { and } \pi_{m}<0 \text {, otherwise } 0 .
$$

Define $\operatorname{ch}(\pi):=\sum_{j} \delta_{j}^{\pi}$ to be the total sign change in $\pi$. For example, if $\pi=1 \overline{3} \overline{2} 2 \overline{3} \overline{1} \overline{1}$, then $\delta^{\pi}=1011001$ and $\operatorname{ch}(\pi)=4$. We begin with the following relationship between the sign change function and the statistics of flag descent, descent and flag major index.

Lemma 17. For each $\pi \in P^{ \pm}(\mathbf{m})$, we have

$$
\begin{aligned}
& \operatorname{fdes}(\pi)=\operatorname{ch}(\pi)+\sum_{\substack{j \in \operatorname{DES}(\pi) \\
\delta_{j}^{\pi}=0}} 2, \\
& \operatorname{des}(\pi)=\lceil\operatorname{ch}(\pi) / 2\rceil+\sum_{\substack{j \in \operatorname{DES}(\pi) \\
\delta_{j}^{\pi}=0}} 1, \\
& \operatorname{fmaj}(\pi)=\sum_{\substack{j \in \operatorname{DESS}(\pi) \\
\delta_{j}^{\pi}=0}} 2 j+\sum_{j=1}^{m} \delta_{j}^{\pi} j .
\end{aligned}
$$

Proof. A descent $j$ of $\pi$ can arise in three cases:

- zero descent: $j=0$ and $\pi_{1}<0$, or

- sign change descent: $\delta_{j}^{\pi}=1$ and $\pi_{j}>\pi_{j+1}$, or

- standard descent: $\delta_{j}^{\pi}=0$ and $\pi_{j}>\pi_{j+1}$.

Regarding sign change descents, consider the partial sums $\sum_{j=k}^{m} \delta_{j}^{\pi}$ of sign changes. By induction on $(m-k)$, we can show that

$$
\sum_{j=k}^{m} \delta_{j}^{\pi}=\chi\left(\pi_{k}<0\right)+2 \mid\{i \geq k: i \text { is a sign change descent of } \pi\} \mid,
$$

from which we obtain (5.5) and (5.6). Also, by (5.8) we have

$$
\begin{aligned}
\sum_{j=1}^{m} \delta_{j}^{\pi} j=\sum_{k=1}^{m} \sum_{j=k}^{m} \delta_{j}^{\pi} & =\sum_{k=1}^{m}\left(\chi\left(\pi_{k}<0\right)+2 \mid\{i \geq k: i \text { is a sign change descent of } \pi\} \mid\right) \\
& =\operatorname{neg}(\pi)+\sum_{k=1}^{m} 2 \mid\{i \geq k: i \text { is a sign change descent of } \pi\} \mid \\
& =\operatorname{neg}(\pi)+\sum_{\substack{j \in \operatorname{DES}(\pi) \\
\delta_{j}^{\pi}=1}} 2 j,
\end{aligned}
$$

which shows (5.7). 
In the following, we deal with the multivariate identities involving flag descents and descents separately. Let $z_{0}, z_{1}, z_{\overline{1}}, z_{2}, z_{\overline{2}}, \ldots$ be a sequence of variables.

5.1. An identity involving flag descents. We introduce the flag version of barred permutations. For $\pi \in P^{ \pm}(\mathbf{m})$ a flag barred permutation on $\pi$ is obtained from $\pi$ by inserting bars such that

- every $j$-th descent space $(1 \leq j \leq m)$ with $\delta_{j}^{\pi}=0$ of $\pi$ receives at least 2 bars;

- the parity of the number of bars in the $j$-th space $(1 \leq j \leq m)$ of $\pi$ has the same parity as $\delta_{j}^{\pi}$.

For example, ||$\overline{1}|\overline{2} 2| 1||$ is a barred permutation on $\pi=\overline{1} \overline{2} 21$ but not a flag barred permutation, the flag barred permutation on $\pi$ with the least number of bars is $\overline{1}|| \overline{2}|2| \mid 1$.

Lemma 18. Let $\mathrm{NDW}_{m}(r)$ be the set of all nondecreasing words of length $m$ with letters from $\{0,1, \ldots, r\}$. For each $b \in \mathrm{NDW}_{m}(r)$ let $\operatorname{tot}(b) \operatorname{tot}^{e}(b), \operatorname{tot}^{o}(b)$ and $\operatorname{odd}(b)$ denote the sum of all letters, the sum of all even letters, the sum of all odd letters and the number of odd letters in $b$, respectively. Then,

$$
\sum_{b \in \mathrm{NDW}_{m}(r)} p^{\operatorname{tot}^{e}(b)} q^{\operatorname{tot}^{o}(b)} z^{\operatorname{odd}(b)}=\sum_{i=0}^{m}(z q)^{i}\left[\begin{array}{c}
\lfloor(r-1) / 2\rfloor+i \\
i
\end{array}\right]_{q^{2}}\left[\begin{array}{c}
\lfloor r / 2\rfloor+m-i \\
m-i
\end{array}\right]_{p^{2}} .
$$

Proof. Let $b=b_{1} \ldots b_{m} \in \mathrm{NDW}_{m}(r)$. Let $1 \leq i_{1}<\cdots<i_{k} \leq m$ (resp. $1 \leq$ $j_{1}<\cdots<j_{l} \leq m$ ) be the sequence of all integers $i$ (resp. $j$ ) such that $b_{i}$ is even (resp. odd). Then, $b$ is completely characterized by the pair $\left(b^{e}, b^{o}\right)$, where $\left.b^{e}:=\left(b_{i_{1}} / 2\right) \ldots b_{i_{k}} / 2\right)$ and $b^{o}:=\left(\left(b_{j_{1}}-1\right) / 2\right) \ldots\left(\left(b_{j_{l}}-1\right) / 2\right)$. Moreover, $\operatorname{tot}^{e}(b)=$ $2 \operatorname{tot}\left(b^{e}\right), \operatorname{tot}^{o}(b)=2 \operatorname{tot}\left(b^{o}\right)+\left|b^{o}\right|$ and $\operatorname{odd}(b)=\left|b^{o}\right|$. Hence, by the interpretation of $q$-binomial coefficients (3.6) we have

$$
\begin{aligned}
& \sum_{b \in \mathrm{NDW}_{m}(r)} p^{\operatorname{tot}^{e}(b)} q^{\operatorname{tot}^{o}(b)} z^{\operatorname{odd}(b)} \\
& =\sum_{i=0}^{m}\left((z q)^{i} \sum_{b^{o} \in \mathrm{NDW}_{i}(\lfloor(r-1) / 2\rfloor)} q^{2 \operatorname{tot}\left(b^{\circ}\right)}\right)\left(\sum_{b^{e} \in \mathrm{NDW}_{m-i}(\lfloor r / 2\rfloor)} p^{2 \operatorname{tot}\left(b^{e}\right)}\right) \\
& =\sum_{i=0}^{m}(z q)^{i}\left[\begin{array}{c}
\lfloor(r-1) / 2\rfloor+i \\
i
\end{array}\right]_{q^{2}}\left[\begin{array}{c}
\lfloor r / 2\rfloor+m-i \\
m-i
\end{array}\right]_{p^{2}},
\end{aligned}
$$

as desired.

Theorem 19. For each $\mathbf{m} \in \mathbb{P}^{n}$ with $m_{1}+\cdots+m_{n}=m$, we have

$$
\begin{gathered}
\frac{\sum_{\pi \in P^{ \pm}(\mathbf{m})} z^{\operatorname{neg}(\pi)} \prod_{\substack{i \in D_{\delta_{i}^{*}=0}(\pi) \\
\delta_{0}}} z_{0}^{2} z_{\pi_{1}}^{2} z_{\pi_{2}}^{2} \cdots z_{\pi_{i}}^{2} \prod_{i=1}^{m} z_{0}^{\delta_{i}^{\pi}} z_{\pi_{1}}^{\delta_{i}^{\pi}} z_{\pi_{2}}^{\delta_{i}^{\pi}} \cdots z_{\pi_{i}}^{\delta_{i}^{\pi}}}{\left(1-z_{0}\right) \prod_{i=1}^{m}\left(1-z_{0}^{2} z_{\pi_{1}}^{2} z_{\pi_{2}}^{2} \cdots z_{\pi_{i}}^{2}\right)} \\
=\sum_{k \geq 0} z_{0}^{k} \prod_{r=1}^{n} \sum_{i=0}^{m_{r}}\left(z z_{\bar{r}}\right)^{i}\left[\begin{array}{c}
\lfloor(k-1) / 2\rfloor+i \\
i
\end{array}\right]_{z_{\bar{r}}^{2}}\left[\begin{array}{c}
\lfloor k / 2\rfloor+m_{r}-i \\
m_{r}-i
\end{array}\right]_{z_{r}^{2}} .
\end{gathered}
$$


In particular, if $z_{r}=z_{\bar{r}}$ for all $r \geq 1$, then

$$
\frac{\sum_{\pi \in P^{ \pm}(\mathbf{m})} \prod_{\substack{i \in \operatorname{DES}(\pi) \\
\delta_{i}^{\pi}=0}} z_{0}^{2} z_{\pi_{1}}^{2} z_{\pi_{2}}^{2} \cdots z_{\pi_{i}}^{2} \prod_{i=1}^{m} z_{0}^{\delta_{i}^{\pi}} z_{\pi_{1}}^{\delta_{i}^{\pi}} z_{\pi_{2}}^{\delta_{i}^{\pi}} \cdots z_{\pi_{i}}^{\delta_{i}^{\pi}}}{\left(1-z_{0}\right) \prod_{i=1}^{m}\left(1-z_{0}^{2} z_{\pi_{1}}^{2} z_{\pi_{2}}^{2} \cdots z_{\pi_{i}}^{2}\right)}=\sum_{k \geq 0} z_{0}^{k} \prod_{r=1}^{n}\left[\begin{array}{c}
m_{r}+k \\
m_{r}
\end{array}\right]_{z_{r}} .
$$

Proof. For each flag barred permutation $\sigma$ on $\pi$ with $b_{i}$ bars in $i$-th space of $\pi$, we define the weight

$$
\mathrm{wt}_{F}(\sigma)=z^{\operatorname{neg}(\pi)} \prod_{i=0}^{m}\left(z_{0} z_{\pi_{1}} \cdots z_{\pi_{i}}\right)^{b_{i}}
$$

Let $F(\mathbf{m})$ be the set of all flag barred permutations on $P^{ \pm}(\mathbf{m})$. As previously, we count $F(\mathbf{m})$ by the weight $\mathrm{wt}_{F}$ twice.

Fix $\pi \in P^{ \pm}(\mathbf{m})$. The flag barred permutation on $\pi$ with the least number of bars, denoted by $\bar{\sigma}$, has the weight $\operatorname{wt}_{F}(\bar{\sigma})=z^{\operatorname{neg}(\pi)} \prod_{\substack{i \in \operatorname{DES}(\pi) \\ \delta_{i}^{\pi}=0}} z_{0}^{2} z_{\pi_{1}}^{2} z_{\pi_{2}}^{2} \cdots z_{\pi_{i}}^{2} \prod_{i=1}^{m} z_{0}^{\delta_{i}^{\pi}}$ $z_{\pi_{1}}^{\delta_{i}^{\pi}} z_{\pi_{2}}^{\delta_{i}^{\pi}} \ldots z_{\pi_{i}}^{\delta_{i}^{\pi}}$. As every flag barred permutation on $\pi$ can be obtained from $\bar{\sigma}$ by further inserting any number of bars in the 0-th space of $\pi$ and an even number of bars in the $i$-th (for $1 \leq i \leq m$ ) space of $\pi$, we see that counting all the flag barred permutations on $\pi$ according to the weight $\mathrm{wt}_{F}$ gives

$$
\frac{z^{\operatorname{neg}(\pi)} \prod_{\substack{i \in \operatorname{DES}(\pi) \\ \delta_{i}^{\pi}=0}} z_{0}^{2} z_{\pi_{1}}^{2} z_{\pi_{2}}^{2} \cdots z_{\pi_{i}}^{2} \prod_{i=1}^{m} z_{0}^{\delta_{i}^{\pi}} z_{\pi_{1}}^{\delta_{i}^{\pi}} z_{\pi_{2}}^{\delta_{i}^{\pi}} \cdots z_{\pi_{i}}^{\delta_{i}^{\pi}}}{\left(1-z_{0}\right) \prod_{i=1}^{m}\left(1-z_{0}^{2} z_{\pi_{1}}^{2} z_{\pi_{2}}^{2} \cdots z_{\pi_{i}}^{2}\right)} .
$$

Therefore, we have that $\sum_{\sigma \in F(\mathbf{m})} \mathrm{wt}_{F}(\sigma)$ equals the left-hand side of (5.9).

Next, for a fixed integer $k \geq 0$, let $F_{k}(\mathbf{m})$ be the set of flag barred permutations in $F(\mathbf{m})$ with $k$ bars. Now, each flag barred permutation from $F_{k}(\mathbf{m})$ can be obtained in two steps. First, we put $k$ bars in one line and insert $m_{r}$ copies of $r$, for $1 \leq r \leq n$, into the spaces between bars. This yields a permutation from $P(\mathbf{m})$ with $k$ bars, which is not necessarily a flag barred permutation. Second, we determine the signs of all the integers and the order of the integers between each pair of two adjacency bars in the resulting object in such a way that it becomes a flag barred permutation. The signs and the orders of integers are unique according to the definition of a flag barred permutation, since

- all integers between two adjacency bars have the same sign and are in increasing order;

- the sign of an integer in the $(j+1)$-th space (from right to left) of the $k+1$ spaces between the $k$ bars is "+" (resp. "-") if $j$ is even (resp. odd).

Thus, each way of inserting in the first step induces one and only one flag barred permutation. Moreover, inserting an integer $r$ in the $(j+1)$-th space (from right to left) of the $k+1$ spaces between the $k$ bars will contribute the factor $z_{r}^{j}$ (resp. $z z_{\bar{r}}^{j}$ ) to the weight of the resulting flag barred permutation if $j$ is even (resp. odd). Therefore, by Lemma 18 we have

$$
\sum_{\sigma \in F_{k}(\mathbf{m})} \mathrm{wt}_{F}(\sigma)=z_{0}^{k} \prod_{r=1}^{n} \sum_{i=0}^{m_{r}}\left(z z_{\bar{r}}\right)^{i}\left[\begin{array}{c}
\lfloor(k-1) / 2\rfloor+i \\
i
\end{array}\right]_{z_{\bar{r}}^{2}}\left[\begin{array}{c}
\lfloor k / 2\rfloor+m_{r}-i \\
m_{r}-i
\end{array}\right]_{z_{r}^{2}} .
$$


Summing over all $k$ and comparing with the fact that $\sum_{\sigma \in F(\mathbf{m})} \mathrm{wt}_{F}(\sigma)$ equals the left-hand side of (5.9), we get (5.9). By setting $z=1, z_{r}=z_{\bar{r}}(1 \leq r \leq n)$ in (5.9) and applying Lemma 9 we obtain (5.10).

Remark 6. For $z=0$, identity (5.9) yields (5.3). Setting $m_{1}=\cdots=m_{n}=1$ in (5.10), we recover Corollary 6.4 in [1]. By (5.5) and (5.7), if we set $z_{0}=t$ and $z_{1}=z_{2}=\cdots=z_{n}=q$ in (5.10), then we obtain

$$
\frac{(1+t) \sum_{\pi \in P^{ \pm}(\mathbf{m})} t^{\mathrm{fdes}(\pi)} q^{\mathrm{fmaj}(\pi)}}{\left(t^{2} ; q^{2}\right)_{m}}=\sum_{k \geq 0} \prod_{r=1}^{n}\left[\begin{array}{c}
m_{r}+k \\
m_{r}
\end{array}\right]_{q} t^{k}
$$

which appears as identity (7.3) in 8 .

\subsection{An identity involving descents.}

Theorem 20. For each $\mathbf{m} \in \mathbb{P}^{n}$ with $m_{1}+\cdots+m_{n}=m$, we have

$$
\begin{aligned}
& \frac{\sum_{\pi \in P^{ \pm}(\mathbf{m})} z^{\operatorname{neg}(\pi)} z_{0}^{\left\lceil\frac{\mathrm{ch}(\pi)}{2}\right\rceil} \prod_{\substack{i \in \text { DES }(\pi) \\
\delta_{i}^{\pi}=0}} z_{0} z_{\pi_{1}}^{2} z_{\pi_{2}}^{2} \cdots z_{\pi_{i}}^{2} \prod_{i=1}^{m} z_{\pi_{1}}^{\delta_{i}^{\pi}} z_{\pi_{2}}^{\delta_{i}^{\pi}} \cdots z_{\pi_{i}}^{\delta_{i}^{\pi}}}{\prod_{i=0}^{m}\left(1-z_{0} z_{\pi_{1}}^{2} z_{\pi_{2}}^{2} \cdots z_{\pi_{i}}^{2}\right)} \\
& =\sum_{k \geq 0} z_{0}^{k} \prod_{r=1}^{n}\left(\sum_{i=0}^{m_{r}}\left(z z_{\bar{r}}\right)^{i}\left[\begin{array}{c}
i+k-1 \\
i
\end{array}\right]_{z_{\bar{r}}^{2}}\left[\begin{array}{c}
m_{r}-i+k \\
m_{r}-i
\end{array}\right]_{z_{r}^{2}}\right) .
\end{aligned}
$$

In particular, if $z_{r}=z_{\bar{r}}$ for all $r \geq 1$, then

$$
\frac{\sum_{\pi \in P^{ \pm}(\mathbf{m})} z_{0}^{\left\lceil\frac{\mathrm{ch}(\pi)}{2}\right\rceil} \prod_{\substack{i \in \mathrm{DES}(\pi) \\
\delta_{i}^{\pi}=0}} z_{0} z_{\pi_{1}}^{2} z_{\pi_{2}}^{2} \cdots z_{\pi_{i}}^{2} \prod_{i=1}^{m} z_{\pi_{1}^{i}}^{\delta_{i}^{\pi}} z_{\pi_{2}}^{\delta_{i}^{\pi}} \cdots z_{\pi_{i}}^{\delta_{i}^{\pi}}}{\prod_{i=0}^{m}\left(1-z_{0} z_{\pi_{1}}^{2} z_{\pi_{2}}^{2} \cdots z_{\pi_{i}}^{2}\right)}=\sum_{k \geq 0} z_{0}^{k} \prod_{r=1}^{n}\left[\begin{array}{c}
m_{r}+2 k \\
m_{r}
\end{array}\right]_{z_{r}} .
$$

Proof. For each barred permutation $\sigma$ on $\pi \in P^{ \pm}(\mathbf{m})$ with $b_{i}$ bars in the $i$-th space of $\pi$, we define the weight

$$
\mathrm{wt}_{B}(\sigma)=z^{\mathrm{neg}(\pi)} \prod_{i=0}^{m}\left(z_{0} z_{\pi_{1}}^{2} \cdots z_{\pi_{i}}^{2}\right)^{b_{i}} \prod_{\pi_{i}<0} z_{\pi_{i}} .
$$

Fix $\pi \in P^{ \pm}(\mathbf{m})$. Then through similar discussion as in the second paragraph of the proof of Theorem 6 we can see that counting all barred permutations on $\pi$ according to the weight $\mathrm{wt}_{B}$ gives

We claim that

$$
\frac{z^{\operatorname{neg}(\pi)} z_{0}^{\operatorname{des}(\pi)} \prod_{i \in \operatorname{DES}(\pi)} z_{\pi_{1}}^{2} \cdots z_{\pi_{i}}^{2} \prod_{\pi_{i}<0} z_{\pi_{i}}}{\prod_{i=0}^{m}\left(1-z_{0} z_{\pi_{1}}^{2} \cdots z_{\pi_{i}}^{2}\right)} .
$$

$$
z_{0}^{\left\lceil\frac{\operatorname{ch}(\pi)}{2}\right\rceil} \prod_{\substack{i \in \operatorname{DES}(\pi) \\ \delta_{i}^{\pi}=0}} z_{0} z_{\pi_{1}}^{2} \cdots z_{\pi_{i}}^{2} \prod_{i=1}^{m} z_{\pi_{1}}^{\delta_{i}^{\pi}} \cdots z_{\pi_{i}}^{\delta_{i}^{\pi}}=z_{0}^{\operatorname{des}(\pi)} \prod_{i \in \operatorname{DES}(\pi)} z_{\pi_{1}}^{2} \cdots z_{\pi_{i}}^{2} \prod_{\pi_{i}<0} z_{\pi_{i}},
$$

from which we see that $\sum_{\sigma \in B(\mathbf{m})} \mathrm{wt}_{B}(\sigma)$ equals the left-hand side of (5.11). Clearly, the degrees of $z_{0}$ on both sides of (5.13) are equal follows from (5.6). To complete 
the proof of our claim, it remains to show that for each $1 \leq i \leq m$, the degrees of $z_{\pi_{i}}$ on both sides of (5.13) are equal. By (5.8), the degree of $z_{\pi_{i}}$ on the left-hand side of (5.13) is

$$
\begin{aligned}
2 \mid\left\{j \geq i: j \in \operatorname{DES}(\pi) \text { and } \delta_{j}^{\pi}=0\right\} \mid & +\sum_{j=i}^{m} \delta_{j}^{\pi} \\
=2\left(\mid\left\{j \geq i: j \in \operatorname{DES}(\pi) \text { and } \delta_{j}^{\pi}=0\right\} \mid\right. & \left.+\mid\left\{j \geq i: j \in \operatorname{DES}(\pi) \text { and } \delta_{j}^{\pi}=1\right\} \mid\right) \\
& \quad+\chi\left(\pi_{i}<0\right)
\end{aligned}
$$

which is exactly the degree of $z_{\pi_{i}}$ on the right-hand side of (5.13). This completes the proof of our claim.

Next, for a fixed integer $k \geq 0$, we can show that

$$
\sum_{\sigma \in B_{k}(\mathbf{m})} \mathrm{wt}_{B}(\sigma)=z_{0}^{k} \prod_{r=1}^{n}\left(\sum_{i=0}^{m_{r}}\left(z z_{\bar{r}}\right)^{i}\left[\begin{array}{c}
m_{r}-i+k \\
m_{r}-i
\end{array}\right]_{z_{r}^{2}}\left[\begin{array}{c}
i+k-1 \\
i
\end{array}\right]_{z_{\bar{r}}^{2}}\right)
$$

by similar discussions as in the proof of Theorem [6. Therefore, $\sum_{\sigma \in B(\mathbf{m})} \mathrm{wt}_{B}(\sigma)$ also equals the right-hand side of (5.11), which finishes the proof of the theorem.

Remark 7. For $z=0$, identity (5.11) yields (5.3). Setting $m_{1}=\cdots=m_{n}=1$ in (5.12), we recover Theorem 6.10 in [1. By (5.6) and (5.7), if we set $z_{0}=t$ and $z_{1}=z_{\overline{1}}=\cdots=z_{n}=z_{\bar{n}}=q$ in (5.11), then we go back to (3.2).

\section{Final REMARKS}

First of all, it would be interesting to find a bijective proof or a $q$-analog of the equidistribution (1.1). Probably, a combinatorial interpretation of recurrence (2.1) in terms of the inversion sequences would help us to understand this equidistribution better.

Recall that a polynomial $f\left(z_{1}, \ldots, z_{m}\right) \in \mathbb{R}\left[z_{1}, \ldots, z_{m}\right]$ is said to be stable, if whenever $\mathfrak{I}\left(z_{i}\right)>0$ for all $i$, then $f$ does not vanish. It is well known that the stability of the multivariate generating functions implies that their univariate counterparts, obtained by diagonalization, have only real roots (see [10, Lemma 2.3]). For each $\mathbf{m} \in \mathbb{P}^{n}$, the three descent polynomials $\sum_{\pi \in P(\mathbf{m})} t^{\operatorname{des}(\pi)}, \sum_{\pi \in P^{ \pm}(\mathbf{m})} t^{\operatorname{des}(\pi)}$ and $\sum_{\pi \in P^{ \pm}(\mathbf{m})} t^{\mathrm{fdes}(\pi)}$ appearing in this paper all have only real roots. It would be interesting to find some multivariate generalizations of the above three descent polynomials, similar to the results for generalized Stirling permutations discovered by Haglund and Visontai [10, which have the nice stable property.

Finally, all the results in this paper remain true if we use the following different ordering:

$$
\{\overline{1}<\overline{2}<\cdots<\bar{n}<\cdots<0<1<2<\cdots<n<\cdots\} .
$$

Moreover, the multivariate identities appearing in Section 5 can be generalized naturally from signed multipermutations to $r$-colored multipermutations for $r \geq 1$.

\section{ACKNOWLEDGEMENT}

The author would like to thank the referee for the valuable comments and suggestions. He was supported by the China Scholarship Council (CSC) for studying abroad. 


\section{REFERENCES}

[1] Matthias Beck and Benjamin Braun, Euler-Mahonian statistics via polyhedral geometry, Adv. Math. 244 (2013), 925-954, DOI 10.1016/j.aim.2013.06.002. MR.3077893

[2] Francesco Brenti, Unimodal, log-concave and Pólya frequency sequences in combinatorics, Mem. Amer. Math. Soc. 81 (1989), no. 413, viii+106, DOI 10.1090/memo/0413. MR.963833 (90d:05014)

[3] Francesco Brenti, q-Eulerian polynomials arising from Coxeter groups, European J. Combin. 15 (1994), no. 5, 417-441, DOI 10.1006/eujc.1994.1046. MR.1292954(95i:05013)

[4] W.Y.C. Chen, A.J.X. Guo, P.L. Guo, H.H.Y. Huang and T.Y.H. Liu, s-inversion Sequences and P-Partitions of type $B$, arXiv:1310.5313.

[5] Chak-On Chow and Ira M. Gessel, On the descent numbers and major indices for the hyperoctahedral group, Adv. in Appl. Math. 38 (2007), no. 3, 275-301, DOI 10.1016/j.aam.2006.07.003. MR2301692 (2008g:05007)

[6] L. Carlitz, A combinatorial property of q-Eulerian numbers, Amer. Math. Monthly 82 (1975), 51-54. MR0366683 (51 \#2930)

[7] Ira Gessel and Richard P. Stanley, Stirling polynomials, J. Combinatorial Theory Ser. A 24 (1978), no. 1, 24-33. MR0462961 (57 \#2926)

[8] Dominique Foata and Guo-Niu Han, Signed words and permutations. III. The MacMahon Verfahren, Sém. Lothar. Combin. 54 (2005/07), Art. B54a, 20. MR2196519 (2007c:05003)

[9] D. Foata and G.-N. Han, The q-series in Combinatorics; Permutation Statistics, Preliminary version, May 5, 2011.

[10] J. Haglund and Mirkó Visontai, Stable multivariate Eulerian polynomials and generalized Stirling permutations, European J. Combin. 33 (2012), no. 4, 477-487, DOI 10.1016/j.ejc.2011.10.007. MR2864433

[11] Percy A. MacMahon, Combinatory analysis, Two volumes (bound as one), Chelsea Publishing Co., New York, 1960. MR.0141605 (25 \#5003)

[12] Pietro Mongelli, Excedances in classical and affine Weyl groups, J. Combin. Theory Ser. A 120 (2013), no. 6, 1216-1234, DOI 10.1016/j.jcta.2013.03.006. MR.3044539

[13] Carla D. Savage and Michael J. Schuster, Ehrhart series of lecture hall polytopes and Eulerian polynomials for inversion sequences, J. Combin. Theory Ser. A 119 (2012), no. 4, 850-870, DOI 10.1016/j.jcta.2011.12.005. MR2881231 (2012m:05052)

[14] C.D. Savage and M. Visontai, The s-Eulerian polynomials have only real roots, Trans. Amer. Math. Soc., to appear (2013).

[15] Rodica Simion, A multi-indexed Sturm sequence of polynomials and unimodality of certain combinatorial sequences, J. Combin. Theory Ser. A 36 (1984), no. 1, 15-22, DOI 10.1016/0097-3165(84)90075-X. MR728500 (85e:05015)

[16] Richard P. Stanley, Ordered structures and partitions, American Mathematical Society, Providence, R.I., 1972. Memoirs of the American Mathematical Society, No. 119. MR0332509 $(48 \# 10836)$

Institut Camille Jordan, Université Claude Bernard Lyon 1, France

Current address: School of Sciences, Jimei University, Xiamen, 361021, People's Republic of China

E-mail address: lin@math.univ-lyon1.fr 